\title{
Martin Winckler: la fiction qui fait approfondir un enjeu de société
}

Jean Martin

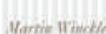

En souvenir d'André

MARTIN WINCKLER

Martin Winckler En souvenir d'André

Paris: POL; 2012

199 p. $26.40 \mathrm{CHF}$

ISBN 978-2-8180-1692-3
J'apprécie les ouvrages de Martin Winckler que j'ai lus, ceux qui traitent de la pratique et de la formation médicales: «La maladie de Sachs», succès de librairie dont un film a été tiré, puis «Les trois médecins» (je ne connais guère ses romans policiers ou ses publications sur les séries télévisées, dont il est un spécialiste).

Le regard qu'il porte sur des aspects de la médecine française - et de ceux qui la pratiquent - retient l'attention, notamment en rapport avec la lenteur avec laquelle elle s'ouvre à des modes de relation soigné-soignant moins inégaux, considérant adéquatement le patient comme un interlocuteur qui a des droits: entre autres le droit de bénéficier, sans avoir à la demander, de toute l'information qui le concerne, dans la véracité et de manière à garantir un consentement informé, et d'avoir accès à son dossier sans entraves. On trouve encore dans les mœurs médicales françaises passablement de paternalisme suffisant et de mandarinat. Ceci alors que dans les textes, légaux (comme la loi dite Kouchner de 2002) ou autres, y compris de l'Ordre national des médecins, le contenu est fort proche de ce qui est actuellement vu, dans les pays plus «partenariaux», comme le cadre fondamental des droits des patients.

«En souvenir d'André» est intitulé roman, comme les ouvrages évoqués ci-dessus. Toutefois, à la différence de ceux-ci dans lesquels l'importance du matériel autobiographique est reconnue, il a plus un caractère de fiction. Extrait d'un interview (les citations sont tirées soit de l'ouvrage lui-même, soit du site web de l'auteur - www.martinwinckler.com - ou de liens à partir de ce site): "C'est clairement un roman. C'est la première fois que je traite un sujet qui ne repose pas lourdement sur mon expérience professionnelle de médecin.» Mais on peut penser que beaucoup de passages sont néanmoins en lien avec l'expérience de l'auteur durant vingt cinq ans, comme généraliste de campagne et comme vacataire dans des consultations hospitalières, où il a toujours été très sensible aux histoires individuelles qui lui étaient confiées.

\section{Lutte contre la douleur et soins palliatifs}

La première partie du livre conte l'histoire du héros quand, jeune médecin, il intègre une unité de lutte contre la douleur en milieu hospitalier. Notamment la confrontation avec les tenants d'une médecine qui voit comme une défaite le constat qu'un malade est perdu, défaite que l'on cherche à cacher ou déguiser - souvent en déléguant à des subalternes la tâche de s'occuper des mourants.

Dans ce contexte, les membres de l'équipe antidouleur cherchent de diverses manières à soulager les derniers jours des patients: «Nous avons débranché les gavages insupportables, les moniteurs stridents, les respirateurs inutiles, les perfusions superflues. Nous avons couché les mourants sur des matelas plus confortables, sous des lumières plus douces, nous leur avons évité les humiliations. Nous avons fait au mieux pour apaiser leur fin.» Il veut pour la fin de vie des «conditions non ignominieuses». Ceci tout en notant un «gaspillage phénoménal»; avec comme par hasard (pages 93 à 98) un passage dévastateur sur les médecins, particulièrement les patrons, corrompus par l'industrie du médicament.

A propos du vécu quotidien: «J'ai appris à apaiser la douleur des autres, à les aider à ne plus être dans la douleur totale, qui empêche de ressentir quoi que ce soit d'autre. Qui empêche de penser. De sourire. D'être présent au monde.» Autour de lui, son action est appréciée; on voit combien elle est nécessaire mais on craint de déroger aux règles, aux routines d'une médecine qui semble estimer que si on souffre bien dans ce monde, on sera mieux accueilli dans l'autre. Pourtant: «Ce que j'avais dans ma sacoche, tous les médecins y avaient accès. Mais beaucoup avaient peur. De quoi? D'être trop puissants? De dépasser leur but? La douleur précipite dans un cercle vicieux. La morphine amorce un cercle vertueux (...). Je n'ai jamais eu peur de trop soulager. Quand la douleur est intolérable, personne ne doit la tolérer.»

Pas de prosélytisme militant, le héros du livre ne tend pas à imposer ses vues ou ses soins mais il entend être à l'écoute des malades, répondre à leur demande. «Je me sentais engagé par le contrat de disponibilité que j'avais passé avec eux.»

\section{Inégalités et incohérences devant la mort prochaine}

C'était une époque donc où le partenariat soignantsoigné était une notion qu'on n'imaginait même pas... «En ce temps-là, le silence et la soumission régnaient en maîtres. Les médecins statuaient souverainement [s'agissant de mettre un terme à la vie d'un patient dont ils jugeaient qu'il n'y avait pas lieu de la prolonger]. Mais lorsqu'un homme ou une 
femme sentaient que leur vie n'était plus que douleur et chagrin, ces mêmes médecins ne trouvaient pas raisonnable leur désir d'y mettre un terme.»

Winckler relève les faveurs que, sans même qu'il soit besoin d'en parler explicitement, on s'accorde mutuellement au sein des élites - et qui se voient à l'approche de la mort comme ailleurs dans la vie médicale et sociétale. «En France, la décision de mourir est violemment connotée à la classe sociale. C'est très facile de mourir comme on veut quand on est dans les hautes couches de la société.» Ces situations le scandalisent, il parle d' «arbitraire idéologique de la corporation et de la classe sociale la plus élevée.» «On ne refusait jamais une ultime injection à un banquier ou à la vieille mère d'un ministre qui se mourait d'un cancer. Mais si la demande venait d'un jeune tétraplégique anonyme assigné à vivre indéfiniment dans un poumon d'acier, il n'était pas pas d'accorder son attention à l'entourage des malades et ses besoins. «L'assistant [accompagnant d'un suicide] reçoit, écoute, ne dit rien, n'intervient pas, ne fait pas d'analyse sauvage ou d'interprétation intempestive ... il est là.»

\section{Accompagner, une tâche qui n'est pas réservée aux soignants}

«Je pensais, depuis longtemps déjà, qu'il n'est pas nécessaire d'être un professionnel pour accompagner celui qui choisit de mourir. Veiller [un malade en fin de vie] fait partie de l'expérience humaine.»

L'auteur parle ici de relation de soins au sens large. On doit pouvoir se faire assister, dit-il, par n'importe qui (en qui on a confiance). Ce qui rappelle le mot du bioéthicien Alex Mauron relevant que le médecin qui donne assistance à un suicide n'agit pas forcément à titre professionnel mais aussi

\section{«C'est très facile de mourir comme on veut quand on est dans les hautes couches de la société.»}

question de l'entendre. Ce garçon était probablement dépressif. Ou manipulé par l'entourage. Ou mal informé des multiples possibilités de survivre dans des conditions acceptables.»

\section{Assistance au suicide}

Le sujet principal autour duquel tourne «En souvenir d'André» est le suicide assisté, interdit en France à ce stade. Récit de la trajectoire d'un médecin qui veut fermement mieux gérer la douleur des malades et voit que, parfois, leur donner le moyen de mourir est une façon de ne pas les abandonner - à vues médicales et humaines, la moins mauvaise issue. Problématique actuelle en France notamment où le prof. Didier Sicard, président d'honneur du Comité consultatif national d'éthique, a remis en décembre dernier un rapport demandé par le Président de la République sur la fin de vie, les soins palliatifs, le suicide assisté, l'euthanasie. S'agissant d'euthanasie, elle est évoquée dans le livre comme une pratique clandestine pas rare, fait qui n'est guère contesté, mais n'a pas été pratiquée par le médecin vieillissant qui raconte sa vie à un jeune interlocuteur (dont l'identité reste floue jusqu'aux dernières pages Winckler met du suspense dans ses œuvres autres que des romans policiers aussi).

Précision donnée dans un interview: «Le suicide assisté n'est pas le seul thème du roman, il y a deux autres questions importantes: l'accompagnement des personnes qui vont mourir passe d'abord par le traitement de la douleur, et ensuite par l'écoute, la possibilité d'entendre des choses que les personnes en fin de vie ne peuvent pas dire à leurs proches, n'ont jamais pu dire auparavant» - cela ne l'empêche comme «confrère en humanité» (à ceci près il est vrai que la prescription d'une dose létale de barbiturique, moyen habituellement employé, n'est possible que par un médecin).

Manifester publiquement à visage découvert? Dans le contexte français, Winckler évoque (à ce stade comme fiction) l'hypothèse d'un «Manifeste des 343 assassins», qui seraient des médecins rendant public qu'ils ont apporté une aide au suicide voire pratiqué l'euthanasie. Faisant écho à celui des 343 «salopes» qui, dans le Nouvel Observateur du 5 avril 1971, déclaraient qu'elles avaient eu un avortement, à une époque où cela restait interdit en France (avant donc la loi Simone Veil de 1975)*.

Désobéissance civile: s'élever contre des lois iniques en entrant au vu de tous dans l'illégalité. Ce peut être le moyen de progresser, notamment quand l'autorité est si mal à l'aise qu'elle renonce à punir. Les femmes ayant déclaré en 1971 des interruptions illégales de grossesse étaient alors passibles de poursuites pénales mais n'ont pas été inquiétées, peutêtre les 343 «assassins» ne le seraient-ils pas?** Occasion de relever que la justice britannique, tout en ayant entamé des démarches contre les proches de plus d'une centaine de personnes parce qu'elles avaient aidé ces dernières à se rendre à Zurich pour y mourir (par Dignitas), n'en a condamné aucune alors que la loi dit qu'une telle aide est punissable. En France, le médecin qui en 2003 a permis le décès euthanasique de Vincent Humbert, tétraplégique et aveugle suite à un accident, n'a finalement pas encouru de sanctions. s'agissant d'assistance au suicide, on ne saurait parler d'assassinat. 